\title{
Compensatory Health Beliefs Relate to Decision-Making Coherence and Health Patterns
}

\author{
BETHANY D. MERILLAT ${ }^{1}$ and CLAUDIA GONZÁLEZ-VALLEJO, Ohio University, Athens, OH, USA.
}

\begin{abstract}
This study tested hypotheses that link cognitive decision-making coherence and health behavioral patterns to the endorsement of compensatory health beliefs (CHBs). Structural equation modeling was used to investigate relationships among the latent variables Compensatory Health Beliefs and 2 other constructs: Decision-Making Coherence (measured by resistance to framing, under/overconfidence, applying decision rules, consistency in risk perception, and resistance to sunk cost bias), and Risk Tendencies with Health Consequences (measured by self-control, the Health Behavior Checklist, and risk perception scales). An online, adult, United States sample-recruited through Amazon.com ${ }^{\circledR}$ 's MechanicalTurk (MTurk)—was assessed. The model described key relationships for the MTurk sample. Low levels of Decision-Making Coherence and Risk Tendencies with Health Consequences were associated with increased endorsement of Compensatory Health Beliefs. Results can help clarify the relationship between health-related cognitions and actions, and impact the design of interventions that rely on the use of the CHB scale.
\end{abstract}

Publication Date: December 2019

https://doi.org/10.18061/ojs.v119i2.6709

OHIO J SCI 119(2):79-91

\section{INTRODUCTION}

Dieters are often constrained by the cognitive effort required in keeping weight loss goals. Indulging in prohibited foods may thus follow simpler appraisals of situations that attempt to compensate one poor behavior with another. A psychological construct pertaining to such substitutions is a compensatory health belief $(C H B)$ (Rabiau et al. 2006). A compensatory health belief, as defined by Rabiau et al. (2006), refers to an individual's "beliefs that the negative effects of an unhealthy behavior can be compensated for, or 'neutralized,' by engaging in another, healthy behavior." The aforementioned authors provide an example: an individual may claim "I can eat this piece of cake now because I will exercise this evening." Knäuper et al. (2004) developed a scale to measure such beliefs, known as the Compensatory Health Beliefs scale (CHB scale). Items on the $\mathrm{CHB}$ scale assess compensatory health beliefs in the categories of substance abuse, eating and sleeping, and stress and weight regulation.

Research using the $\mathrm{CHB}$ scale has documented relationships among health beliefs, health behavior, self-regulation, and health outcomes (Abraham et al. 2000). Increased scores in the CHB scale have been found to be associated with higher body mass index (BMI), increased risky-health and weightregulating behaviors (e.g., dieting), and decreased

${ }^{1}$ Address correspondence to Bethany D. Merillat, Ohio University, 11224 Seasons Drive, Apt. 2, Toledo, OH 43615. Email: bl872911@ohio.edu health-related self-efficacy-confidence in being able to self-regulate (Knäuper et al. 2004). In addition, items in the $\mathrm{CHB}$ scale regarding glucose testing in diabetic patients (e.g., "Testing my glucose regularly is not that important if I eat the same things every day") were found to be associated with poorer selfcare behaviors (Rabiau et al. 2009). Smoking-specific items in the CHB scale were also found to account for an individual's readiness to stop smoking even after controlling for levels of conscientiousness (known as a level of self-discipline) (Radtke et al. 2011). Further, smoking-specific items were also found to be significantly and negatively associated with the intention to stop smoking; this is above and beyond other well-known predictors of health behavior change such as risk perception, intention, and action planning (e.g., Schwarzer's (2008) Health Action Process Approach) (Radtke and Scholz 2012). More recently, scores in the CHB scale were found to be predictive of vaccination behavior, in addition to the intentions to vaccinate (Ernsting et al. 2012).

In terms of psychometric properties of the $\mathrm{CHB}$ scale, several studies have documented evidence of internal and test-retest reliability for the overall scale (de Nooijer et al. 2009; Kaklamanou et al. 2013), whereas the subscales appear to be less consistent (de Nooijer et al. 2009). In terms of predicting theoretical

(C) 2019 Merillat and González-Vallejo. This article is published under a Creative Commons Attribution 4.0 International License

( https://creativecommons.org/licenses/by/4.0/) 
meaningful relationships of $\mathrm{CHB}$ scores with other criteria (i.e., assessments of criterion validity), the research is extensive but heterogeneous. In a sample of 381 undergraduate students at McGill University, Knäuper et al. (2004) found the CHB scores were positively correlated with responses to the Irrational Health Belief Scale (IHBS) (Christensen et al. 1999) and with health-related risk behaviors (Thompson et al. 1999). These results give evidence of convergent validity because higher endorsement of compensatory health beliefs related to less healthy behaviors as theorized by the authors of the measure. On the other hand, CHB scores were un related to socially desirable responding (M-C SDS, Marlowe-Crowne Social Desirability Scale) (Crowne and Marlowe 1960), and to race/ethnicity, age, or major in the university. These latter findings support discriminant validity as the $\mathrm{CHB}$ construct is not theoretically expected to relate to a person's age or other such peripheral factors.

The current project further advances construct validity of the $\mathrm{CHB}$ scale by formally incorporating cognitive processes that affect decision-making in various domains. As suggested by Rabiau et al. (2006), endorsing compensatory health beliefs leads to poor health outcomes to the extent that (1) the compensatory behavior does not fully compensate for the negative effects and/or (2) the individual fails to follow through with the intended behavior. This implies that there is a level of inconsistency between intentions and actions that pertains to a basic level of decision-making incoherence. Furthermore, a general lack of self-control, and/or erroneous perceptions that actions leading to poor outcomes are less risky, may also relate to endorsing compensatory health beliefs (Knäuper et al. 2004). A goal of this project was to explore the extent to which endorsing compensatory bealth beliefs relates to decision-making variables pertaining to thought coherence and self-control. Key constructs were introduced that are hypothesized to relate to compensatory health beliefs. A formal model was presented to test the expected relationships.

\section{Decision-Making Coherence}

In the decision-making literature, there are 2 major ways of assessing the ability of humans to make good decisions: coherence and correspondence standards (Hammond 1996). Coherence refers to the use of logical rules that lead to consistent thoughts and behaviors; correspondence refers to the agreement between a judgment and ecological criteria (e.g., does a judgment accurately predict an event in the world). Decision-making coherence is the cornerstone of rational decision-making models (Savage 1954; Edwards 1955) which assume that individuals maximize their personal expected utility by adhering to basic principles of logic called axioms (Schoemaker 1982; Plous 1993). An example is the axiom of transitivity which states that people should have a coherent set of preferences: if they prefer $A$ to $\mathrm{B}$, and $\mathrm{B}$ to $\mathrm{C}$, they should prefer $\mathrm{A}$ to $\mathrm{C}$. In the case of correspondence, the predictive ability of a judge is evaluated in light of how that prediction is reflected in the ecological outcome (e.g., if the forecast says it will rain, does it rain) (Dunwoody 2009).

Early work on compensatory health beliefs suggested that coherence is linked to health and health behaviors. For example, a study by Knäuper et al. (2005) found that poor adherence to self-set dieting goals undermined weight loss success. This suggests that individuals who can follow rules consistently may have higher levels of internal coherence, and be better able to achieve a goal.

In a separate literature, a wide range of experiments have shown that humans tend to violate the axioms of utility theory (Tversky 1969; Kahneman and Tversky 1979; Budescu and Weiss 1987). Changes in problem wording (framing) affect decisionmaking consistency. A famous example of framing was presented by Tversky and Kahneman (1981), known as the Asian disease scenario. When asked to choose which option participants would prefer in response to an outbreak of an Asian disease anticipated to kill 600 people, $72 \%$ of respondents preferred Program A (200 people will be saved) over Program B (a one-third probability that 600 people will be saved, and two-thirds probability that no people will be saved). But when asked to choose between Program C (400 people will die) and Program D (a one-third probability that nobody will die, and a two-third probability that 600 people will die), $78 \%$ chose Program D over Program C. The inconsistency results in the change of risk preference for identical problems. That is, a selection of the sure gain in the first scenario (saving 200 lives) is identical to selecting that 400 individuals would die in the second scenario; however, the second choice problem does not result in a preference for the identical sure loss. Given these findings, individuals who are more prone to commit such decision-making errors are more likely to endorse compensatory health beliefs. 
Framing effects and other decision-making errors are pervasive, but there are individual differences. For example, a study by LeBoeuf and Shafir (2003) examined whether or not framing effects could be avoided or reduced. Results showed that naturally thoughtful individuals (i.e., those who enjoy thinking and scored high on the Need for Cognition Scale) (Cacioppo et al. 1984) had greater consistency despite framing effects. This possibility was tested in the current study, but need for cognition did not relate to other variables and was not discussed further. The current study was thus narrowed to include classic decision-making errors such as framing, overconfidence (i.e., having greater confidence relative to accuracy of predictions), sunk cost bias (i.e., following a losing decision based on past costs), consistency in risk perceptions, and applying rules consistently. Further descriptions of these measures are found in the Methods section.

Self-control is another psychological aspect found to relate to problematic health behaviors. Selfcontrol refers to taking a course of action that yields a more positive outcome in the long term than in the short term (Baumeister and Heatherton 1996). While Baumeister et al. (1998) did not measure compensatory health beliefs, their work suggests that the effort required to exert self-control in one task can diminish the ability to resist a temptation. For example, a study by Monson et al. (2008) found that depleting self-control (e.g., presenting dieters with a tempting chocolate cookie vs. a "diet" cookie) led to increased compensatory health beliefs formation. Namely, compensatory beliefs were more prevalent in dieters faced with a temptation (chocolate cookies) than faced with reduced-fat cookies. The current study uses an individual level measure of self-control to explore its relationship with the endorsing of compensatory health beliefs.

A compensatory health belief model presented by Rabiau et al. (2006) suggested that when faced with a temptation, risk adaptation is one of the ways in which an individual can resolve the conflict. Knäuper et al. (2004) reported that higher CHB scores were significantly related to health risk behaviors (e.g., smoking, lower consumption of healthy foods, and decreased physical activity). Further focus was on assessing the relationships of risk perceptions and the endorsement of compensatory health beliefs. Individuals with low risk perception (e.g., they see dangerous situations as less risky) may be less inclined to endorse compensatory health beliefs because they don't view risky actions (e.g., drinking alcohol) as harmful - and therefore see no need to take compensatory actions.

As discussed earlier, prior research has demonstrated relationship between $\mathrm{CHB}$ scores and health outcomes such as BMI (Thompson et al. 1999), intentions to stop smoking (Radtke and Scholz 2012), and vaccination behavior (Ernsting et al. 2012). It was reasoned that these observed relations may reflect a more general trend: individuals endorsing compensatory health beliefs having lifestyle patterns that negatively impact health. These patterns may be, for example, not eating a balanced diet and/or not seeing the doctor for regular checkups (Vickers et al. 1990).

\section{A Model of the Relations of Decision- Making Coherence, Health Risks, and Compensatory Health Beliefs}

Astructural equation model (SEM) was developed based on the theoretical features of a model proposed by Rabiau et al. (2006) and the earlier described theoretical considerations. The full model is found in Lavins (2013), which first tested all measurement models linking latent variables to observed indicators. The current study presents a reduced model that reproduced the observed hypothesized relationships. Details of the model tests and statistics are found in the Results section. As in conventional SEM analysis, latent variables appear as ellipses, whereas measured (indicator) variables appear in rectangles. Arrows denote directional paths relating measures and constructs with weights indicating the level of strength of the relationships. Error terms are shown in circles for measured variables.

The model in Fig. 1 assumes 3 latent variables: Risk Tendencies with Health Consequences, Compensatory Health Beliefs, and Decision-Making Coherence. The construct of Risk Tendencies with Health Consequences is assumed to encompass many aspects of risks that ultimately affect health. These pertain to (1) behaving more impulsively in various situations - as described by the notion of self-control-which can affect survival risks, or (2) perceiving things that one indulges in (e.g., smoking) as less risky than they are, or (3) engaging in activities that are risky. Thus, Risk Tendencies with Health Consequences is assumed to be measured by scales of Self-Control, Risk Perception, and the 
Health Behaviors Checklist (HBCL) (Vickers et al. 1990). The HBCL contains various items assessing health activities, such as speeding while driving, that increase (or decrease) mortality risks. Details of these measures are found in the Methods section.

The construct of Decision-Making Coherence is also assumed to impact Compensatory Health Beliefs, with higher coherence resulting in lower beliefs. This latent variable is measured by several subscales of the Adult Decision-Making Competence index (ADMC) (Bruine de Bruin et al. 2007), which address consistency in thinking, judging, and making choices.

The construct of Compensatory Health Beliefs is measured by the Compensatory Health Beliefs scale (Knäuper et al. 2004) and the Likelihoodscale (Lavins 2013) which measures an individual's likelihood of engaging in a compensatory activity. Details about each of the scales appear in the Methods section.

The model in Fig. 1 uses parceling in order to simplify the model and show key relationships among the constructs. All of the scales in the model demonstrated good internal consistency; thus, following recommendations by Matsunaga (2008), the items were combined prior to creating the structural equation model in Fig. 1. Clearly, several other models are possible-including some that do not use parceling. The test was restricted to the model in Fig. 1 because the main purpose of the current investigation was to provide an initial demonstration that the constructs pertaining to health patterns and coherence in thinking relate to compensatory health beliefs (see Lavins (2013) for work on the evaluation of other models).

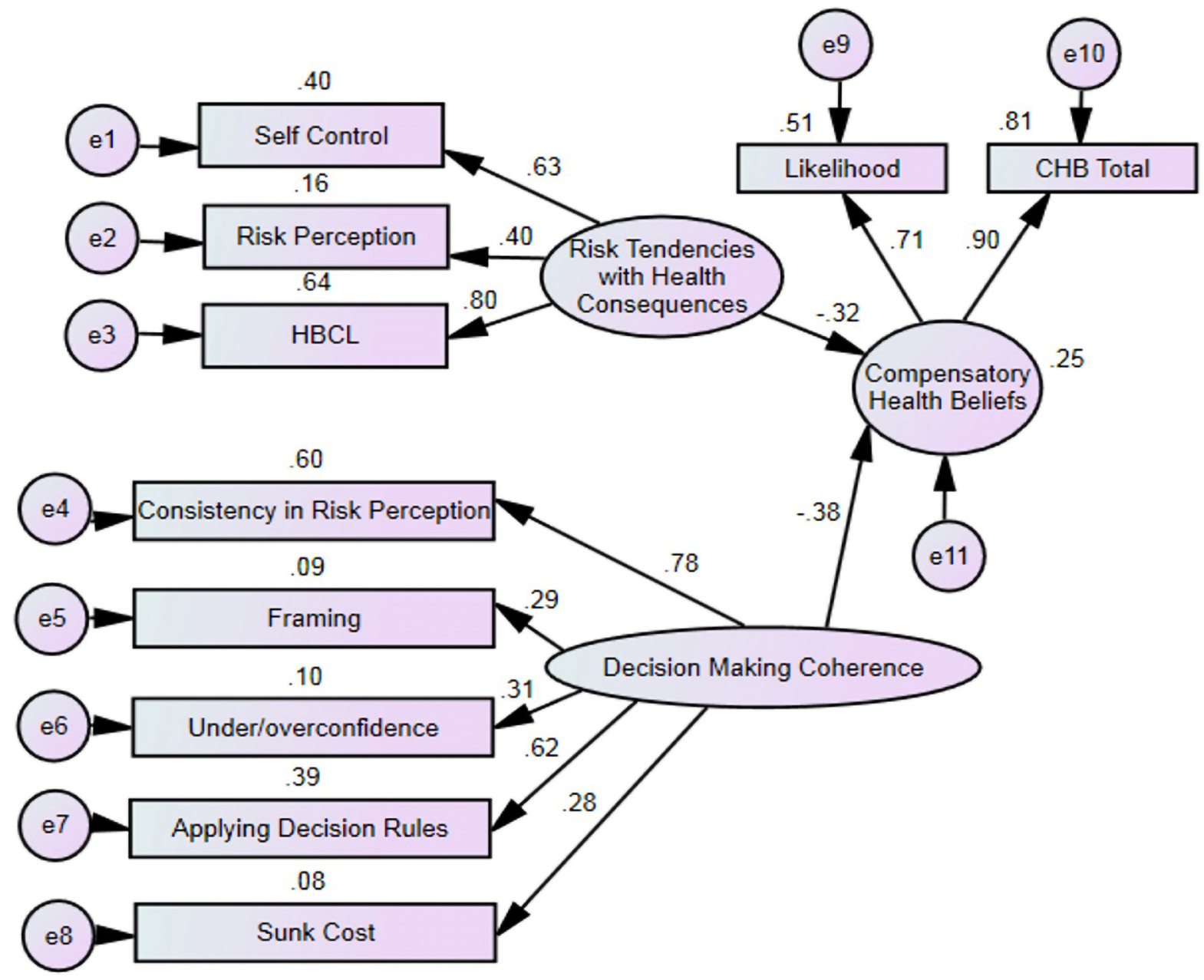

FIGURE 1. Structural equation model relating Risk Tendencies with Health Consequences and Decision-Making Coherence to Compensatory Health Beliefs. Values reported are standardized regression weights. Numbers above the rectangles represent the variance of the variable indicated by the box. All path coefficients are significant at the $p<0.01$ level. Note that both Framing and Sunk Cost in the figure refer to measures of resistance to the Framing and Sunk Cost Biases, thus higher values mean greater Decision-Making Coherence. 


\section{METHODS AND MATERIALS Participants and Procedures}

Participants were 217 individuals recruited through Amazon.com ${ }^{\circledR}$ 's Mechanical Turk (MTurk). A review of MTurk by Buhrmester et al. (2011) suggested that the tool provides high-quality data at least as reliable as could be obtained through traditional recruitment methods. The MTurk samples are also more diverse than the average college sample. While some have questioned the motivations of individuals willing to work for such low wages, a review of MTurk found that $69.6 \%$ of United States workers claimed that they participate because MTurk is a fruitful way to spend time (as opposed to watching TV) (Paolacci et al. 2010; Horton et al. 2011; Chandler and Kapelner 2013).

Participants were adults older than 18, living in the United States (according to MTurk's filters) who signed up for the study on www.mturk.com (see Results section for demographics). Recommendations for power estimates for selected levels of degrees of freedom $(d f)$ and sample size were used to determine the sample size for testing the present structural equation model (MacCallum et al. x1996). Using 51 degrees of freedom to reach a power of $80 \%$, the current study required a sample of 200.

Using MTurk, participants were directed to the Qualtrics ${ }^{\circledR}$ (2019) website where they completed an online survey lasting between 45 and 60 minutes, and received compensation ( $\$ 0.90$ for participating) as a thank you for their time. An option on Qualtrics prevented participants from taking the survey twice from the same IP address. All measures used in this study can be found online at the website of the Society for Judgment and Decision Making (SJDM 2019) (http://www.sjdm.org), and specifically at:

http://www.sjdm.org/dmidi/Adult___Decision_Making_Competence.html and http://www.sjdm.org/dmidi/files/AdultDMCwithoutPathIndep.pdf

\section{Measurements of Compensatory Health Beliefs}

The CHB scale (Knäuper et al. 2004). The $\mathrm{CHB}$ scale was comprised of 17 items that ask participants to read each sentence and respond by indicating how much they agree or disagree on a 5 -point scale $(0=$ "totally disagree" to 4 = "totally agree"). The scale was scored by creating an overall total score for the participant (potential range 0 to 68) with higher scores indicating higher belief in (reliance on) compensatory health behaviors. The 4 subscales were: (1) substance use-6 items concerning behaviors that could compensate for alcohol and coffee consumption and smoking, (2) eating/sleeping-4 items concerning behaviors to compensate for lack of sleep/poor eating choices, (3) stress-4 items concerning behavior to compensate for stress, and (4) weight regulation-3 items concerning behaviors to compensate for excessive calorie intake.

The Likeliboodscale. This scale was developed and tested by Lavins (2013) in an earlier study, similar to the work of Kaklamanou et al. (2013) in their analysis of the CHB scale. The Likelihood scale consists of the same 17 items of the $\mathrm{CHB}$ scale, but the questions ask the likelihood of engaging in a compensatory activity rather than a belief. The scale ranges from 0 to 1.00 . Scores are calculated by creating an average of the participant's reported likelihood of engaging in each of the 17 items, with higher scores indicating a greater likelihood of engaging in the behavior.

\section{Measures of Decision-Making Coherence} The Adult Decision-Making Competence index (ADMC) (Bruine de Bruin et al. 2007). The ADMC assesses how well individuals make decisions across 7 domains. Five subscales most related to the construct under study were used: Resistance to Framing, Under/ overconfidence, Applying Decision Rules, Consistency in Risk Perception, and Resistance to Sunk Cost. For example, a question on sunk cost bias describes a scenario in which the participant is asked to imagine having a large meal at a restaurant and then ordering a big dessert, but, after the first few bites of the dessert, feels full. The participant is then asked whether she/ he would continue consuming the dessert. Asserting a greater likelihood of consuming the dessert demonstrates the sunk cost bias, because a coherent decision maker should carry out actions based on the expected utility of consuming the dessert rather than on the already committed cost. These subscales were also chosen for having at least moderate reliability in past studies (Bruine de Bruin et al. 2007).

The ADMC was scored according to the authors' instructions. Resistance to framing was calculated as the absolute difference between ratings of related frames. The under/overconfidence scores resulted from computing 1 minus the absolute difference between the mean confidence and the percent correct. Applying decision rules was the percent of correct answers to that set of items. Consistency in 
risk perception was the percent of consistent risk judgments. Resistance to sunk cost was the average rating across items that measured sunk cost bias (Table 1) (Bruine de Bruin et al. 2007). Higher scores in the ADMC indicate overall greater consistency in making decisions.

\section{Measure of Self-Control}

The Self-Control scale (Tangney et al. 2004). This 36-item scale is a self-report measure of degree of control. Participants respond to questions such as, "I am good at resisting temptation," on a 1 to 5 scale ( 1 = "not at all" to 5 = "very much"). Other items include, "I wish I had more self-discipline" and "I spend too much money." The scale total is scored by creating a sum of responses to the 36 items (potential range of 36 to 180), with higher scores indicating a greater degree of self-control.

\section{Measures of Risk Perception}

The Risk scale (Blais and Weber 2006). This 30-item, 2-part, scale assesses risk perceptions and risk behavior in 5 content domains (6 items per subscale): financial (investing \& gambling), health/ safety, recreational, ethical, and social decisions. In part I, respondents rate the likelihood on a 1 to 7 scale ( 1 = "extremely unlikely" to 7 = "extremely likely") that they would engage in each activity (e.g., "bungeejumping off a tall bridge," "riding a motorcycle without a helmet," and "sunbathing without sunscreen"). Part II assess the respondents' perceptions of the magnitude of the risks of the activities judged in part I on a 1 to 7 scale ( 1 = "not at all risky" to 7 = "extremely risky"). In the current study, risk perceptions were focused on and thus used part II only.

This risk perception scale is a different measure from the Consistency in Risk Perception of the ADMC. The risk perception scale from Blais and Weber (2006) focuses on assessment of both actual self-reported risk taking and attitudes toward risk taking (e.g., the degree to which an activity is perceived as risky). By contrast, the ADMC focuses on the calculation of risks from the perspective of making consistent rational choices among gambles.

\section{Measures of Health Behaviors}

The Health Behaviors Checklist (HBCL) (Vickers et al. 1990). The HBCL is a 40-item survey which measures health in 2 general areas: preventive health and risk-taking behaviors. Participants indicate their agreement with statements describing their typical behavior on a 1 to 5 scale ( $1=$ "strongly disagree" to 5 = "strongly agree") in 4 health-related domains: wellness maintenance (e.g., "I take vitamins"), accident control (e.g., "I have a first aid kit in my home"), traffic related (e.g., "I speed while driving"), and the use of potentially harmful substances (e.g., "I don't drink alcohol"). The overall scale is scored by creating an average of the participant's responses across the 27 items used for the subscales; higher scores indicate healthier behaviors. A recent analysis found reliability estimates of 0.63 to $0.79(M=0.69)$ across the 4 scales (Madhavan 2004).

\section{Demographic Questions}

Demographic questions were also included assessing the participant's height and weight (used to calculate body mass index (BMI)) (CDC 2015), age, gender, year in school, employment status, income, ethnicity, and dieting status. BMI is calculated as the individual's weight divided by height squared $\left(\mathrm{lb} / \mathrm{in}^{2}\right)$ multiplied by a conversion factor of 703 .

\section{Demographics}

\section{RESULTS}

Participants $(n=217)$ reported they were predominantly female (69.59\%), not currently dieting (76.50\%), employed (52.53\%), and currently students $(10.14 \%)$. The samplewaslargely Caucasian (79.26\%), but also included African Americans (7.37\%), Hispanics (4.15\%), Asians (2.76\%), and Native Americans (2.76\%). The mean age was 38.62 $(S D=13.42)$, and average BMI for this group was $27.12(S D=7.35)$. Additionally, $81.1 \%$ of the sample reported an annual income of less than $\$ 59,000$ per year. In terms of education, the majority of the participants were not in college, with $45.16 \%$ reporting they were either graduated, pursuing higher education, or not currently enrolled in classes ("other"). Of those reporting being in academic status (10.14\%), 3.7\% were seniors, followed by juniors (2.3\%), sophomores (1.84\%), and freshman (1.84\%).

\section{Descriptive Statistics of Measures}

Table 1 displays descriptive statistics for the scales included in this study. The table shows ranges along with average scores and Cronbach's a reliability.

The overall reliability of the $\mathrm{CHB}$ scale was good (Cronbach's $a=0.80)$. Reliability for the Likelihood scale was also good (Cronbach's $a=0.85$ ), correlating 
Table 1

MTurk descriptive statistics for the measures $(n=217)$

\begin{tabular}{lcccccc}
\hline \hline Measures & Mean & Min & Max & SD & Cronbach's a & No. of items \\
\hline CHB total $^{\text {a }}$ & 29.07 & 7 & 62 & 9.42 & 0.80 & 17 \\
Likelihood avg. & 0.43 & 0.02 & 0.88 & 0.16 & 0.85 & 17 \\
HBCL b avg. $^{3.40}$ & 2.11 & 4.7 & 0.52 & 0.83 & 27 \\
Self-Control total & 121.67 & 74 & 180 & 20.90 & 0.92 & 36 \\
Risk Perception total & 143.27 & 76 & 210 & 22.24 & 0.88 & 30 \\
ADMC subscales & & & & & & \\
$\quad$ Resistance to Framing & 4.01 & 2.29 & 4.93 & 0.47 & 0.60 & 14 \\
$\quad$ Under/overconfidence & 0.90 & 0.62 & 1 & 0.07 & 0.77 & 34 \\
$\quad$ Applying Decision Rules & 0.64 & 0 & 1 & 0.25 & 0.76 & 10 \\
$\quad$ Consist. in Risk Perception & 0.73 & 0.3 & 0.9 & 0.11 & 0.66 & 20 \\
$\quad$ Resistance to Sunk Cost Bias & 4.25 & 2.4 & 6 & 0.69 & 0.54 & 10 \\
\hline
\end{tabular}

${ }^{a} \mathrm{CHB}$ had a possible range from 0 to 68 .

${ }^{\mathrm{b}}$ Health Behaviors Checklist.

${ }^{c}$ All Adult Decision-Making Competence (ADMC) subscales are scored so higher numbers reflect better performance.

strongly with total scores on the $\mathrm{CHB}$ scale $(r(217)=0.64, p<0.01)$. The CHB scale appeared to perform better for the substance use, eating/sleeping, and stress subscales — but worse for weight regulation. Alpha levels in the current sample were generally higher for the subscales than in either the original Knäuper et al. (2004) or de Nooijer et al. (2009) studies.

As seen in Table 1, measures of self-control, risk perception, and HBCL all had good reliability (Cronbach's $a>0.8$ ). In terms of the ADMC scale, responses in our sample were similar to those found by Bruine de Bruin et al. (2007) indicating acceptable internal consistency reliability (Cronbach's a range $=0.60$ to 0.77 ), with the exception of sunk cost bias measure (Cronbach's $a=0.54$ ). The scales making up the entire ADMC scale contain item scoring that varies depending on the specific bias the items try to demonstrate. Indeed, a typical score is computed by assessing responses to 2 items, thus items in these scales are not measures of the construct as is typical of Likert-type scales. This consideration was also a factor in using parceling in the structural equation model tested.

\section{Correlations}

Table 2 presents the correlations of all of the measures presented. As seen in the table, the CHB scale was significantly correlated (all values of $p<0.05)$ with the Likelihood scale, Self-Control,
Risk Taking, HBCL, Risk Perception, and all ADMC subscales-except Resistance to Framing. BMI was not correlated with any of the measures and thus was not incorporated in the model in Fig. 1.

\section{Model Evaluation: Variables Predictive of Compensatory Health Beliefs}

Structural equation modeling (SEM) tests were performed using AMOS ${ }^{\mathrm{TM}}$ (Arbuckle 2006) and path coefficients were examined for statistical significance. While SEM is similar to other common quantitative methods_-such as correlation, multiple regression, and analysis of variance (ANOVA) - it is unique in its capacity to estimate and test relationships among constructs (Weston and Gore 2006). Whereas traditional linear models represent constructs with only 1 measure, SEM may have several measures and allows for error in measurement to be modeled. SEM analysis evaluates multiple test statistics and fit indices to determine if a model accurately represents relationships among constructs and observed measures. The result is that the analysis is concerned with fit, or the extent to which the prediction and the observed pattern match, and thus looks for non-significant differences between predictions and observations.

The scales used in the model were examined for outliers, skewness, and kurtosis, in addition to reliability (Mardia 1974). All of the variables appeared 
Table 2

Correlations of all of the measures presented

(MTurk correlations, $n=217$ )

\begin{tabular}{|c|c|c|c|c|c|c|c|c|c|c|c|c|}
\hline Measures & 1 & 2 & 3 & 4 & 5 & 6 & 7 & 8 & 9 & 10 & 11 & 12 \\
\hline 1. $\mathrm{BMI}$ & - & & & & & & & & & & & \\
\hline 2. CHB scale & -0.114 & - & & & & & & & & & & \\
\hline 3. Likelihood & -0.093 & $0.639^{*}$ & - & & & & & & & & & \\
\hline $\begin{array}{l}\text { 4. Resistance to } \\
\text { Framing }^{1}\end{array}$ & 0.049 & -0.057 & -0.041 & - & & & & & & & & \\
\hline $\begin{array}{l}\text { 5. Under/ } \\
\text { overconfidence }^{1}\end{array}$ & $0.209^{*}$ & $-0.226^{*}$ & -0.136 & 0.008 & - & & & & & & & \\
\hline $\begin{array}{l}\text { 6. Applying } \\
\text { Decision Rules }{ }^{1}\end{array}$ & 0.115 & -0.171 & -0.006 & $0.301^{*}$ & 0.171 & - & & & & & & \\
\hline $\begin{array}{l}\text { 7. Consistency in } \\
\text { Risk Perception }{ }^{1}\end{array}$ & 0.079 & $-0.282^{*}$ & 0.211 & 0.183 & $0.257^{*}$ & $0.478^{*}$ & - & & & & & \\
\hline $\begin{array}{l}\text { 8. Resistance to } \\
\text { Sunk Cost Bias }{ }^{1}\end{array}$ & -0.006 & -0.061 & 0.011 & 0.103 & 0.037 & 0.139 & $0.249^{*}$ & - & & & & \\
\hline $\begin{array}{l}\text { 9. HBCL average } \\
(27)\end{array}$ & -0.035 & -0.150 & 0.184 & -0.09 & 0.062 & 0.211 & -0.038 & -0.031 & - & & & \\
\hline 10. Self-Control & -0.158 & $-0.240^{*}$ & $0.236^{*}$ & -0.01 & 0.087 & -0.029 & 0.102 & 0.009 & $0.507^{*}$ & - & & \\
\hline 11. Risk Perception & 0.055 & -0.142 & 0.188 & -0.009 & 0.026 & $0.250^{*}$ & 0.024 & 0.044 & $0.340^{*}$ & 0.197 & - & \\
\hline 12. ADMC_Z_total & 0.150 & $-0.268^{*}$ & -0.129 & $0.536^{*}$ & $0.495^{*}$ & $0.702^{*}$ & $0.729^{*}$ & $0.514^{*}$ & -0.104 & 0.053 & -0.056 & - \\
\hline
\end{tabular}

${ }^{*} p<0.001$

${ }^{1}$ ADMC subscales

to be symmetrically distributed. Further, there were no missing data. Results showed that the model in Fig. 1 had $\chi^{2}(33, N=217)=62.51, p=0.001$; had a good level of root mean square error of approximation, RMSEA $=0.064,90 \% C I[0.039,0.088]$; and a CFI of 0.916 -suggesting that the model worked well according to the recommendations in Schreiber et al. (2006). It was noted that although the $\chi^{2}$ is significant, this is not of great concern because good fits are difficult to attain with large sample sizes. The power of this test statistics increases rapidly with increases in sample size, making even small discrepancies between predictions and observations significant (Henson 2006). Thus, finding a nonsignificant $\chi^{2}$ may be unlikely even if the model were a close fit to the observed data (Weston and Gore 2006). In addition, examination of standardized residuals also suggested adequate model fit.

The paths found in Fig. 1 show standardized regression weights. The weights were all greater than 0.31 and significant at the $p<0.01$ level, displaying fairly strong relationships among the variables. Checks on the estimated weights showed no squared multiple correlations greater than 1 , and no negative error variances. Fig. 1 also shows variance on top of each measured indicator.

Standardized and unstandardized coefficients for the model in Fig. 1, and the relationships among the variables, are summarized in Table 3 . All the relationships were significant at the $p<0.01$ level.

As observed in Fig. 1 and Table 3, all measurement models show positive coefficients for paths linking the measures (scales) to the latent variables. Furthermore, the assumed latent variable relationships showed the expected negative paths between positive aspects of health and risk perceptions and compensatory health beliefs. That is, individuals having healthier lifestyles, better self-control, and proper perceptions of risks have fewer compensatory health beliefs. Similarly, higher levels of decision-making coherence lead to fewer compensatory health beliefs. 


\section{DISCUSSION}

Findings from this study offer new insight into the mechanisms that may work with, or contribute to, the endorsement of compensatory health beliefs. In terms of psychometrics, this study demonstrated-along with the previous study by de Nooijer et al. (2009) — that the overall $\mathrm{CHB}$ scale is reliable. Further expanding on past research, this current study demonstrated that compensatory health beliefs were negatively related to decision-making coherence and were also negatively related to tendencies that support good health. In combination, this study suggests that individuals who lack decision-making coherence when analyzing decision problems, and who also have poor self-care behaviors, are more likely to endorse compensatory health beliefs. Note that the model tested assumes directional paths stemming from the constructs of Decision-Making Coherence to Compensatory Health Beliefs; thesame is true for the construct of Risk Tendencies with Health Consequences. As is true with all structural equation models, the direction of the paths imply causality; however, strict causal tests are not possible because manipulation of variables following a true experimental paradigm cannot be performed. That is, it is not possible to experimentally lower or increase health behaviors, for example, in order to study its effect on the endorsing of compensatory health beliefs. Longitudinal studies, however, may provide additional evidence for the specified paths described here. A longitudinal clinical trial, for instance, using an intervention to promote health behaviors may also measure compensatory health beliefs and show changes over time for both. The current results thus serve as starting hypotheses to be tested in future works.

With regards to risk tendencies with health consequences, one of the primary findings was its inverse relationship with compensatory health beliefs. This shows that a general pattern of dampened risk perception of risky activities, as well as behaving in manners that can decrease one's well-being (e.g., lack of care for one's health), predict beliefs of compensating bad actions for good ones. Thus, to the extent that healthier habits lead to healthier

Table 3

Standardized and unstandardized coefficients for the final model in Fig. $1(n=217)$

\begin{tabular}{lllll}
\hline \hline Latent variables & Latent variables & $\boldsymbol{\beta}^{*}$ & $\mathbf{B}$ & SE \\
\hline $\begin{array}{l}\text { Risk Tendencies with Health } \\
\text { Consequences }\end{array}$ & Compensatory Health Beliefs & -0.32 & -0.21 & 0.06 \\
Decision-Making Coherence & Compensatory Health Beliefs & -0.38 & -10.38 & 3.40
\end{tabular}

\begin{tabular}{clllc} 
Latent variables & Observed indicator scale & $\boldsymbol{\beta}^{*}$ & B & SE \\
\hline $\begin{array}{c}\text { Risk Tendencies with Health } \\
\text { Consequences }\end{array}$ & Self-Control & 0.63 & 1.00 & - \\
" & Risk Perception & 0.40 & 0.68 & 0.15 \\
& HBCL & 0.80 & 0.03 & 0.01 \\
Decision-Making Coherence & Consistency in Risk Perception ${ }^{1}$ & 0.78 & 2.48 & 0.68 \\
& Resistance to Framing ${ }^{1}$ & 0.29 & 0.93 & 0.36 \\
" & Under/overconfidence ${ }^{1}$ & 0.31 & 1.00 & - \\
$"$ & Applying Decision Rules ${ }^{1}$ & 0.62 & 1.98 & 0.56 \\
" & Resistance to Sunk Cost Bias ${ }^{1}$ & 0.28 & 0.89 & 0.34 \\
Compensatory Health Beliefs & Likelihood scale & 0.71 & 0.23 & 0.05 \\
& CHB scale & 0.90 & 1.00 & - \\
\hline
\end{tabular}

*All standardized coefficients are significant at the alpha 0.01 level.

${ }^{1}$ Measures are part of the Adult Decision-Making Competence scale (ADMC). 
routines, people need fewer compensatory health beliefs to justify their behavior. This result is also consistent with the theory espoused by Rabiau et al. (2006), who hypothesized that compensatory health beliefs would be formed as the easiest of 3 options (i.e., resist the temptation, modify perceptions, or form compensatory health beliefs) to reduce cognitive dissonance associated with health-desire conflicts. The current results show that the endorsement of compensatory health beliefs is the result of other lifestyle patterns and perceptions that indicate poorer health. It is not clear, however, that creating compensatory health beliefs - as an active process of denial_may also induce poor health habits.

Future studies may test this additional possibility with an experimental paradigm rather than a correlational study. One possibility is to produce cognitive dissonance in the laboratory and assess the creation of compensatory health beliefs, along with behavioral measures, that may support healthy habits. For example, a study on eating behavior may both induce cognitive dissonance regarding eating and assess the creation of compensatory beliefs. This proposed study could also test the It was demonstrated bealth beliefs were negatively related to decision-making coberence and were also negatively related to tendencies that support good health. In combination, this study suggests that individuals who lack decisionmaking coberence when analyzing decision problems, and who also have poor selfcare behaviors, are more likely to endorse compensatory bealth beliefs.
Making Competence (ADMC) subscales_-Under/ overconfidence, Applying Decision Rules, and Consistency in Risk Perception-showed moderate levels of correlation with scores on the CHB scale, suggesting the several interesting conclusions below.

With regards to Under/overconfidence, people who were less calibrated (e.g., were certain that they were right, when they were actually wrong) scored higher in the $\mathrm{CHB}$ scale. Overconfident individuals may endorse compensatory health belief statements because they are confident that they can indulge in a temptation and correct it later. To the extent that their ability to accurately predict their own behavior is compromised by their overconfidence, these individuals may be more likely to indulge but not appropriately compensate.

Another component of the ADMC that correlated with scores in the CHB scale was Applying Decision Rules. High scores on Applying Decision Rules indicate being able to accurately follow rules to correctly select the right option. It is possible that people who are competent in this task score lower in the $\mathrm{CHB}$ scale because they have a better understanding of "health rules;" they understand that selection of a healthy vs. a less-healthy snack as a gift for participating in the study (this would be a proxy for measuring healthy actions). At a later time, the study could further assess the endorsement of compensatory health beliefs and new food selections. Thus, future experimental studies are needed to further explore directional hypotheses, with a focus not just on endorsement, but measurement of the creation and use of compensatory health beliefs by participants.

Another new and important finding in the current study concerns the relationship between decision-making coherence and compensatory health beliefs. As hypothesized, results showed a fairly strong negative path between these 2 latent factors, suggesting that individuals who show consistency in the decisions they make across multiple domains are less likely to endorse compensatory health beliefs. Additionally, 3 of the Adult Decision- engaging in compensatory health behaviors (of the ones found in the $\mathrm{CHB}$ scale) will undermine, rather than support, their ultimate health goals.

Consistency in Risk Perception (e.g., not perceiving events happening in the near future to be significantly more risky than those in the distant future) also correlated with scores in the CHB scale. Past research has shown that individuals often fail to take preventive measures related to their healthuntil confronted with a problem-because the costs (e.g., avoiding sweets) seem too high and the rewards (e.g., good health in 40 years) are far and distant (Slovic et al. 1984). Thus, individuals who accurately gauge the seriousness of present and future health risks may be less likely to endorse compensatory health beliefs because they know that the short-term reward (e.g., pleasure from smoking) will undermine their long-term health goals. 


\section{Summary}

The goal of this study was to propose and test a structural equation model that assumed relationships among 3 constructs that, in combination, advance understanding of factors related to healthy behaviors.

Results showed that there are individual-level tendencies pertaining to self-control, risk perceptions, and health patterns that describe a general construct of risk taking that affects health. Similarly, there are individual differences, at the level of decisionmaking coherence, which include several aspects of a person's adherence to normative/logical principles of thinking. In combination, these latent variables predict the extent to which individuals endorse compensatory health beliefs-demonstrating that these beliefs are multi-dimensionally determined. More precisely, the structural equation model showed that compensatory health beliefs were predicted not only from patterns of risk perceptions and habits that have consequences to one's health, but also from patterns of thinking coherently.

Given the increasing usage of the $\mathrm{CHB}$ scale in health research, it is important to have additional understanding of its construct validity; the current work advances this understanding. This current study is cross-sectional, and thus limited in terms of the causal relations the model affords; however, it presents interesting hypotheses regarding the thought processes that may be at the base of poor health habits that are reflected in compensatory health beliefs. Hence, future studies may focus on enhancing decision-making coherence and assessing its effect on actual compensatory behaviors over time.

\section{Limitations}

This study proposed and tested a structural equation model developed using the approach of parceling. This approach has advantages as well as disadvantages as described by Matsunaga (2008). It is possible for other models to reproduce the variance-covariance structure of the data better, but parceling in the current setting was reasonable and the conclusions follow the theoretical considerations presented. Other models were tested by the first author and are found in Lavins (2013).

\section{ACKNOWLEDGEMENTS}

This work was supported by the Ohio University Graduate Student Senate Original Work Research Award: $12 X 217$.

\section{LITERATURE CITED}

Abraham C, Norman P, Conner M. 2000. Towards a psychology of health-related behavior change. In: Norman P, Abraham $\mathrm{C}$, Conner M, editors. Understanding and changing health behavior: from health beliefs to self-regulation. Amsterdam (NL): Harwood Academic Publishers. Chapter 14. p. 343-369.

Arbuckle JL. 2006. Amos ${ }^{\mathrm{TM}} 7.0$ user's guide. Chicago (IL): Marketing Department, SPSS Inc. 583 p.

ISBN-13: 978-1-56827-386-0.

Baumeister RF, Bratslavsky E, Muraven M, Tice DM. 1998. Ego depletion: is the active self a limited resource? J Pers Soc Psychol. 74(5):1252-1265.

https://doi.org/10.1037//0022-3514.74.5.1252

Baumeister RF, Heatherton TF. 1996. Self-regulation failure: an overview. Psychol Inq. 7(1):1-15. https://doi.org/10.1207/s15327965pli0701_1

Blais AR, Weber EU. 2006. A domain-specific risk-taking (DOSPERT) scale for adult populations. Judgm Decis Mak. $1(1): 33-47$.

Bruine de Bruin W, Parker AM, Fischhoff B. 2007. Individual differences in adult decision-making competence. J Pers Soc Psychol. 92(5):938-956. https://doi.org/10.1037/0022-3514.92.5.938

Budescu DV, Weiss W. 1987. Reflection of transitive and intransitive preferences: a test of prospect theory. Organ Behav Hum Dec. 39(2):184-202. https://doi.org/10.1016/0749-5978(87)90037-9

Buhrmester M, Kwang T, Gosling SD. 2011. Amazon's Mechanical Turk: a new source of inexpensive, yet high-quality, data? Perspect Psychol Sci. 6(1):3-5. https://doi.org/10.1177/1745691610393980

Cacioppo JT, Petty RE, Kao CF. 1984. The efficient assessment of need for cognition. J Pers Assess. 48(3):306-307. https://doi.org/10.1207/s15327752jpa4803_13

[CDC] Centers for Disease Control and Prevention. 2015. Atlanta (GA). Body mass index (BMI); 2015 May 15 [accessed 2019 Nov 26]; [1 screen]. https://www.cdc.gov/healthyweight/assessing/bmi/index.html

Chandler D, Kapelner A. 2013. Breaking monotony with meaning: motivation in crowdsourcing markets. J Econ Behav Organ. 90:123-133.

https://doi.org/10.1016/j.jebo.2013.03.003

Christensen AJ, Moran PJ, Wiebe JS. 1999. Assessment of irrational health beliefs: relation to health practices and medical regime adherence. Health Psychol. 18(2):169-176. https://doi.org/10.1037//0278-6133.18.2.169

Crowne DP, Marlowe D. 1960. A new scale of social desirability independent of psychopathology. J Consult Psychol. 24(4):349-354. https://doi.org/10.1037/h0047358

de Nooijer J, Puijk-Hekman S, van Assema P. 2009. The compensatory health beliefs scale: psychometric properties of a cross-culturally adapted scale for use in the Netherlands. Health Educ Res. 24(5):811-817. https://doi.org/10.1093/her/cyp016 
Dunwoody PT. 2009. Theories of truth as assessment criteria in judgment and decision making. Judgm Decis Mak. 4(2):116-125.

http://journal.sjdm.org/ccd/ccd.pdf

Edwards W. 1955. The prediction of decisions among bets. J Exp Psychol. 50(3):201-214.

https://doi.org/10.1037/h0041692

Ernsting A, Schwarzer R, Lippke S, Schneider M. 2012. "I do not need a flu shot because I lead a healthy lifestyle": compensatory health beliefs make vaccination less likely. J Health Psychol. 18(6):825-836. https://doi.org/10.1177/1359105312455076

Hammond KR. 1996. Human judgment and social policy: irreducible uncertainty, inevitable error, unavoidable injustice. Oxford: Oxford University Press. 436 p.

ISBN-13: 978-0195143270.

Henson RK. 2006. Effect-size measures and meta-analytic thinking in counseling psychology research. Couns Psychol. 34(5):601-629.

https://doi.org/10.1177/0011000005283558

Horton JJ, Rand DG, Zeckhauser RJ. 2011. The online laboratory: conducting experiments in a real labor market. Exp Econ. 14(3):399-425. https://doi.org/10.1007/s10683-011-9273-9

Kahneman D, Tversky A. 1979. Prospect theory: an analysis of decision under risk. Econometrica. 47(2):263-291. https://www.jstor.org/stable/1914185

Kaklamanou D, Armitage CJ, Jones CR. 2013. A further look into compensatory health beliefs: a think aloud study. Brit J Health Psych. 18(1):139-154. https://doi.org/10.1111/j.2044-8287.2012.02097.x

Knäuper B, Cheema S, Rabiau M, Borten O. 2005. Self-set dieting rules: adherence and prediction of weight loss success. Appetite. 44(3):283-288.

https://doi.org/10.1016/j.appet.2005.01.008

Knäuper B, Rabiau M, Cohen O, Patriciu N. 2004. Compensatory health beliefs: scale development and psychometric properties. Psychol Health. 19(5):607-624. https://doi.org/10.1080/0887044042000196737

Lavins BD. 2013. Testing the compensatory health belief scale: the role of cognitive factors and their relationship to health outcomes [master's thesis]. [Athens $(\mathrm{OH})$ ]: Ohio University. http://rave.ohiolink.edu/etdc/view?acc_num=ohiou1367609821

LeBoeuf RA, Shafir E. 2003. Deep thoughts and shallow frames: on the susceptibility to framing effects. J Behave Decis Making. 16(2):77-92.

https://doi.org/10.1002/bdm.433

MacCallum RC, Browne MW, Sugawara HM. 1996. Power analysis and determination of sample size for covariance structure modeling. Psychol Methods. 1(2):130-149. https://doi.org/10.1037/1082-989X.1.2.130

Madhavan P. 2004. Assessment of the psychometric properties of the facets of conscientiousness. NAm J Psychol. 6(2):309-326.

Mardia KV. 1974. Applications of some measures of multivariate skewness and kurtosis in testing normality and robustness studies. Sankhyā Ser B. 36(2):115-128. https://www.jstor.org/stable/25051892
Matsunaga M. 2008. Item parceling in structural equation modeling: a primer. Commun Meth Meas. 2(4):260-293. https://doi.org/10.1080/19312450802458935

Monson E, Knäuper B, Knonick I. 2008. Food temptations spontaneously elicit compensatory beliefs in dieters. McGill Sci Undergrad Res J. 3(1):42-45. https://issuu.com/msurj/docs/msurjvol3iss 1

Paolacci G, Chandler J, Ipeirotis PG. 2010. Running experiments on Amazon Mechanical Turk. Judgm Decis Mak. 5(5):411-419. https://EconPapers.repec.org/RePEc:jdm:journl:v:5:y:2010:i:5:p:411-419

Plous S. 1993. The psychology of judgment and decision making. New York (NY): McGraw-Hill. 302 p. ISBN-13: 978-0070504776.

Qualtrics. 2019. Software version: 2012 of the Qualtrics Research Suite. Provo (UT): Qualtrics. https://www.qualtrics.com

Rabiau M, Knäuper B, Miquelon P. 2006. The eternal quest for optimal balance between maximizing pleasure and minimizing harm: the compensatory health beliefs model. Brit J Health Psych. 11(1):139-153. https://doi.org/10.1348/135910705X52237

Rabiau M, Knäuper B, Nguyen TK, Sufrategui M, Polychronakos C. 2009. Compensatory beliefs about glucose testing are associated with low adherence to treatment and poor metabolic control in adolescents with type 1 diabetes. Health Educ Res. 24(5):890-896.

https://doi.org/10.1093/her/cyp032

Radtke T, Scholz U. 2012. "Enjoy a delicious cake today and eat healthily tomorrow": compensatory health beliefs and their impact on health. Eur Health Psychol. 14(2):37-40. https://doi.org/10.5167/uzh-97762

Radtke T, Scholz U, Keller R, Knäuper B, Hornung R. 2011. Smoking-specific compensatory health beliefs and the readiness to stop smoking in adolescents. Brit J Health Psych. 16(3):610-625. https://doi.org/10.1348/2044-8287.002001

Savage LJ. 1954. The foundations of statistics. New York (NY): John Wiley \& Sons. 294 p.

https://doi.org/10.1002/nav.3800010316 ISBN-13: 978-0486623498 [2nd rev. edition]

Schreiber JB, Nora A, Stage FK, Barlow EA, King J. 2006. Reporting structural equation modeling and confirmatory factor analysis results: a review. J Educ Res. 99(6):323-338. https://doi.org/10.3200/JOER.99.6.323-338

Schwarzer R. 2008. Modeling health behavior change: how to predict and modify the adoption and maintenance of health behaviors. Appl Psychol-Int Rev. 57(1):1-29. https://doi.org/10.1111/j.1464-0597.2007.00325.x

Schoemaker PJH. 1982. The expected utility model: its variants, purposes, evidence and limitations. J Econ Lit. 20(2):529-563.

https://www.jstor.org/stable/2724488

[SJDM] The Society for Judgment and Decision Making [homepage, http://www.sjdm.org]. 2019. Adult-decision making competence (ADMC) [applicable portion]; 2007 [accessed 2019 Dec 28]; [1 page, about 2 screens]. http://www.sjdm.org/dmidi/Adult___Decision_Making_Competence.html 
Slovic P, Fischoff B, Lichtenstein S. 1984. Behavioral decision theory perspectives on risk and safety. Acta Psychol. 56(1-3):183-203. https://doi.org/10.1016/0001-6918(84)90018-0

Tangney JP, Baumeister RF, Boone AL. 2004. High self-control predicts good adjustment, less pathology, better grades, and interpersonal success. J Pers. 72(2):271-324. https://doi.org/10.1111/j.0022-3506.2004.00263.x

Thompson BL, Nelson DE, Caldwell B, Harris JR. 1999. Assessment of health risk behaviors: a tool to inform consumers, providers, health care organizations, and purchasers. Am J Prev Med. 16(1):48-59. https://doi.org/10.1016/S0749-3797(98)00091-9
Tversky A. 1969. Intransitivity of preferences. Psychol Rev. 76(1):31-48. https://doi.org/10.1037/h0026750

Tversky A, Kahneman D. 1981. The framing of decisions and the psychology of choice. Science. 211(4481):453-458. https://doi.org/10.1126/science. 7455683

Vickers RR Jr, Conway TL, Hervig LK. 1990. Demonstration of replicable dimensions of health behaviors. Prev Med. 19(4):377-401. https://doi.org/10.1016/0091-7435(90)90037-K

Weston R, Gore PA Jr. 2006. A brief guide to structural equation modeling. Couns Psychol. 34(5):719-751.

https://doi.org/10.1177/0011000006286345 